\title{
Ultrathin fluorescent layers for monitoring the axial resolution in confocal and two-photon fluorescence microscopy
}

\author{
M. SCHRADER, ${ }^{*}$ U. G. HOFMANN† \& S. W. HELL* \\ *High Resolution Optical Microscopy Group, Max-Planck-Institut for Biophysical Chemistry, \\ 37070 Göttingen, Germany \\ †Åbo Akademi, Institutionen för Fysikalisk Kemi, 20500 Turku, Finland and Ludwig- \\ Maximilians-Universität, Lehrstuhl für Angewandte Physik, 80799 München, Germany
}

Key words. 4Pi, axial resolution, confocal microscopy, fluorescence, monolayer, point-spread-function, polymerizable lipid, two-photon excitation, z-response.

\section{Summary}

Monomolecular films of polymerized dimethyl-bis[pentacosadiinoic-oxyethyl] ammonium bromide (EDIPAB) provide one- and two-photon excited fluorescence that is sufficiently high to quantify the axial resolution of 3-D fluorescence microscopes. When scanned along the optical axis, the fluorescence of these layers is bright enough to allow online observation of the axial response of these microscopes, thus facilitating alignment and fluorescence throughput control. The layers can be used for directly measuring and monitoring the axial response of $4 \mathrm{Pi}$-confocal microscopes, as well as for their initial alignment and phase adjustment. The proposed technique has the potential to supersede the conventional technique of calculating the derivative of the axial edges of a thick fluorescent layer. Coverslips with EDIPAB-layers can be used as substrates for the cultivation of cells.

\section{Introduction}

The axial sectioning capability and the intrinsic axial resolution are key properties of confocal and multiphoton fluorescence microscopes (Wilson \& Sheppard, 1984; Denk et al., 1990; Wilson, 1990). Unfortunately, the laws of diffraction dictate that the axial resolution be more responsive to aberrations than its lateral counterpart (Born \& Wolf, 1993). However, this phenomenon makes the observation of the axial resolution very sensitive for testing the performance of confocal and multiphoton microscopes. Axial resolution is best quantified by the $z$ response which is the response to an ultrathin fluorescence

Correspondence to: S. W. Hell. E-mail: SHell@gwdg.de. Present address of U. G. Hofmann: California Institute of Technology, Division of Biology 216-76, Pasadena, CA 91125, U.S.A. layer when scanned along the optical axis:

$$
I\left(z^{\prime}\right)=C \int_{0}^{\infty} \int_{0}^{2 \pi} h_{\mathrm{ill}}^{n}(r, z) h_{\mathrm{det}}(r, z) r \mathrm{~d} \phi \mathrm{d} r
$$

$h^{n}{ }_{\text {ill }}$ denotes the $n$th power of the illumination point-spreadfunction (PSF) with $n=1,2 \ldots$ for 1-, 2-, and higher order multiphoton excitation, respectively, $h_{\text {det }}$ is the detection PSF accounting for an (optional) detection pinhole. The fullwidth-at-half-maximum (FWHM) of the z-response is a measure of the axial resolution of the microscope. Moreover, the $z$-response is able to reveal aberrations of practical confocal microscopes (Wilson \& Sheppard, 1984; Wilson \& Carlini, 1989; Sheppard \& Cogswell, 1991; Hell et al., 1993), most notably spherical aberrations which are manifested as a pronounced asymmetry in the response. Therefore, a direct method for the online investigation of the axial resolution would be of great help. The possibility of monitoring the axial resolution of a 3-D microscope would facilitate the implementation of novel 3-D imaging methods and allow a rapid evaluation of their sectioning capabilities.

Standard procedures hitherto comprise the measurement of the derivative of the response to an axial edge produced by a thick fluorescent layer and the scanning of a plane mirror along the optic axis. Both methods allow only an indirect view of the actual axial resolution of fluorescent planes. Whereas the mirror approach does not measure the fluorescence $z$-response, establishing the derivative from the axial edge response is susceptible to noise. In some cases, for example when using rhodamine, it can be compromised by fluorophore sedimentation on the coverslip.

In principle, the axial edge response of the fluorescent layer contains all the information, but in practice it is hard to assess the extent of (spherical) aberrations from the undifferentiated edge response. The measurement of the 
axial resolution with fluorescent beads, although viable, might be affected by the finite bead size, thus necessitating off-line image restoration procedures (Van der Voort \& Strasters, 1995). Beads of acceptable size tend to display a weak signal or be clustered in the specimen. Other methods such as the pulsed-laser-fluorophore-deposition (PLFD)effect (Hell et al., 1995) require production of the layer by nonlinear optical effects and cannot be used in conjunction with a biological specimen. In this paper, we propose another alternative with significant potential to overcome these problems. We provide evidence that monomolecular films of the polymerized diacetylenic lipid dimethyl-bis[pentacosadiinoic-oxyethyl] ammonium bromide are promising candidates for precise and simple axial resolution quantification in fluorescence microscopy.

\section{Materials and methods}

Dimethyl-bis[pentacosadiinoic-oxyethyl] ammonium bromide (EDIPAB), a diacetylenic polymerizable two-chain lipid (Fig. 1), was synthesized following published procedures (Hupfer et al., 1983). Ultrathin monomolecular films were produced by spreading monomers of the molecule out of chloroform on the air-water interface of a water subphase (Milli-Q-System, Millipore, Molsheim, France) of a thermostatized filmbalance at $16^{\circ} \mathrm{C}$ (accessible surface: $450 \times 150 \mathrm{~mm}^{2}$, KSV 5000, KSV Instruments, Espoo, Finland) standing in a class 100 cleanroom hood. The film was compressed up to a final lateral pressure of $27 \mathrm{mN} \mathrm{m}^{-1}$ and then polymerized (leading to a crystalline packing of $\approx 0.50 \mathrm{~nm}^{2}$ per molecule). UV polymerization was carried out with a $\mathrm{Hg}$ lamp at a wavelength of $310 \mathrm{~nm}$ for about
$3 \mathrm{~min}$. After polymerization the films were transferred onto microscope coverslips of standard $(170 \mu \mathrm{m})$ thickness using the standard Langmuir-Blodgett technique (Ulmann, 1991). For that purpose the coverslips were pulled, from being totally submersed, vertically through the air-water interface at a constant speed of about $10 \mathrm{~mm} \mathrm{~min}^{-1}$. The maximum thickness of these layers can be specified by atomic force microscopy (data not shown) to $<3 \mathrm{~nm}$, therefore they can be regarded as infinitely thin compared with the typical axial extent of the effective PSF of the microscope.

Polydiacetylene monolayers exhibit a strong intrinsic fluorescence parallel to the polymer backbone as already described for other polydiacetylenes (Göbel et al., 1987; Moers et al., 1994). Figure 2 shows the emission spectrum of the polymerized film measured directly on the coverslip using a mercury lamp, an illumination bandpass filter $(450-490 \mathrm{~nm})$, a beamsplitter at $510 \mathrm{~nm}$ and a detection longpass at $520 \mathrm{~nm}$. Owing to the positively charged head of the molecule the polymer is strongly bound to the coverslip and withstands cleaning and washing with water and standard detergents.

\section{Experiments}

We placed a drop of immersion oil between two coverslips, one of which was covered with a monomolecular layer of EDIPAB. Next, the coverslips were pressed to obtain a homogeneous layer of immersion oil. Having sandwiched the fluorescent layer between the coverslips we recorded axial and lateral images. Figure 3(a) is an $x y$ image of the EDIPAB-layer as recorded in a single-photon excitation

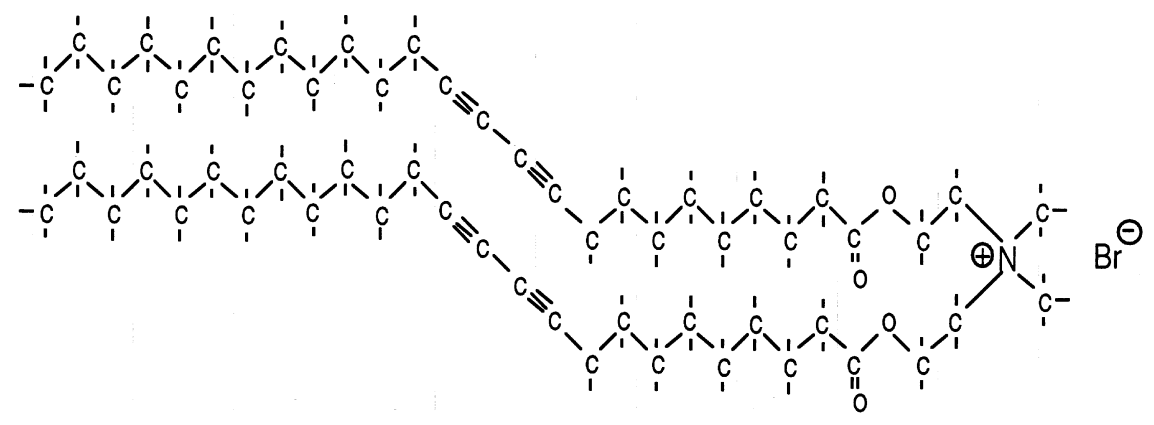<smiles>[2H]CC#CC#CCPCC#CC#CCP</smiles>

Fig. 1. Structural scheme of the lipid EDIPAB and its UV polymerization. 


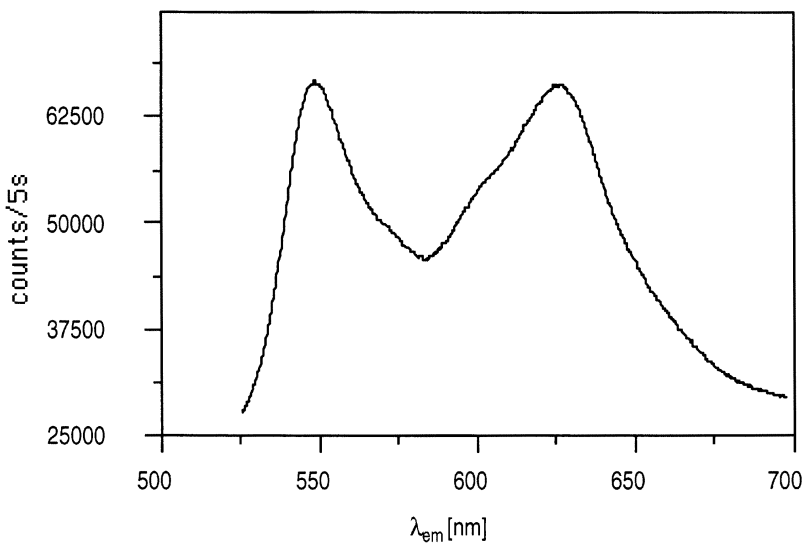

Fig. 2. Fluorescence emission spectrum of EDIPAB polymerized on water and transferred to a microscope coverslip.

confocal microscope (Zeiss, LSM 410). The excitation wavelength was $488 \mathrm{~nm}$; the light was linearly polarized. The image dimensions are $50 \times 50 \mu \mathrm{m}, 512 \times 512$ pixel. The pixel dwell time was $1.4 \mu \mathrm{s}$ and the illumination power $\approx 8 \mu \mathrm{W}$. Figure 3(b) shows an $x z$ image along the line indicated in Fig. 3(a). Figure 3(c) displays a profile of the intensity along the optical axis (three lines averaged). We further note that efficient excitation at $514 \mathrm{~nm}$ and $532 \mathrm{~nm}$ was also possible.

Two-photon excitation images (Fig. 4a,b) were recorded in an object scanning confocal fluorescence microscope (Schrader et al., 1996) by illuminating with a train of 130 fs pulses as provided by a mode-locked Ti : sapphire laser (Mira 900F, Coherent). The excitation wavelength was $810 \mathrm{~nm}$ and the laser light was linearly polarized. Scanning was accomplished using a precise piezoelectric stage (Melles Griot, Cambridge, U.K.) at a fixed pixel dwell time of $2 \mathrm{~ms}$. The fluorescence light passed a dichroic mirror and $2 \mathrm{~mm}$ of Schott blue glass (BG39). The detector was an avalanche photodiode (EG \& G, SPCM130) with a quantum efficiency of $60-70 \%$ in the red regime. Our microscope was slightly confocalized as the back-projected diameter of the pinhole corresponded to about the size of the Airy disk calculated at the fluorescence wavelength. The numerical aperture of the lenses (Leitz, PlanaPo 100x) was 1.4 oil immersion. Figure $4(\mathrm{a}, \mathrm{b})$ were recorded with an average power of $\approx 250 \mu \mathrm{W}$ at the focus.

Figures 3(a) and 4(a) reveal that the film is not uniform; rather it forms crystalline domains which appear bright and dark to the viewer. The latter is due partly to the dichroism of these layers, i.e. the absorption depends on the orientation of the polarization, but within a particular domain the molecules are uniformly orientated. The sizes of the domains depend on the parameters during polymerization and vary from a couple of micrometres, as the example of Fig. 4a, to a couple of hundred micrometres (Göbel, 1989). For the $z$-response measurement a larger crystalline domain of uniform brightness has to be preselected prior to
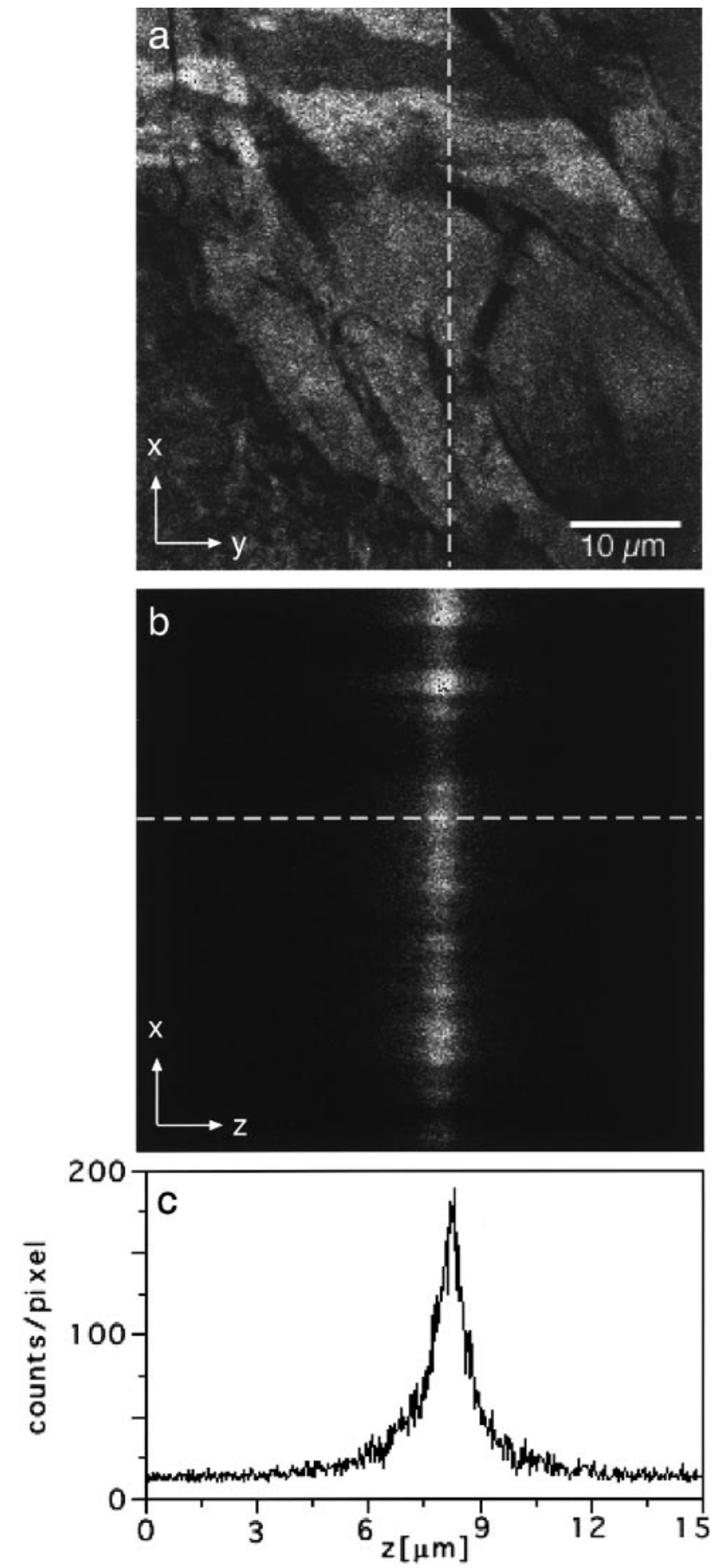

Fig. 3. (a) Confocal one-photon-excitation $x y$ image showing an area of $50 \times 50 \mu \mathrm{m}$; (b) $x z$ image through the area shown in (a); (c) intensity profile through the $x z$ image (laterally averaged across $300 \mathrm{~nm}$ ). The excitation wavelength was $488 \mathrm{~nm}$, pixel dwell time was approximately $1 \cdot 4 \mu \mathrm{s}$.

axial resolution determination. The examples of Fig. 3(a) and $4(\mathrm{a})$ are representative for the manufacturing batch, but we also observed individual coverslips with uniform domains $30 \times 30 \mu \mathrm{m}$ in size. 

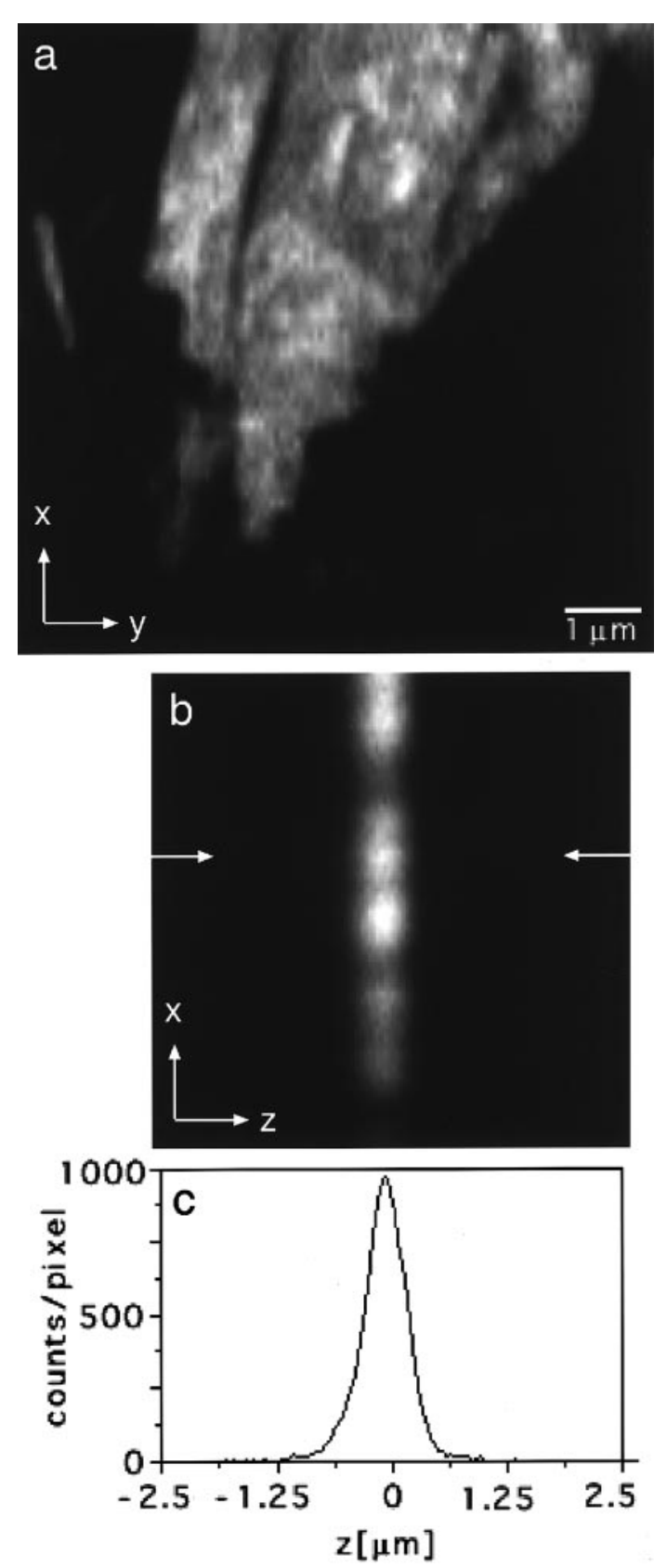

Fig. 4. (a) Confocal two-photon excitation $x y$ image showing part of a single domain of the EDIPAB film; (b) $x z$ image of a domain of the EDIPAB film; (c) intensity profile along the optic axis showing a $z$-response (pixel dwell time $2 \mathrm{~ms}$, axial pixel size $25 \mathrm{~nm}$, no averaging). The excitation wavelength was $810 \mathrm{~nm}$.

The structure of the polymeric domains of EDIPAB has been described before, along with their strong one-photon fluorescence capability and the polarization dependence of their excitation (Tillmann et al., 1994). Our experiments revealed the efficient two-photon excitation of these layers. We did not aim at establishing the two-photon cross-section, but we can give an idea of their relative brightness. In a confocal arrangement rejecting about 50-70\% of the fluorescence at the pinhole, we recorded 500 counts per ms when illuminating the same point of the layer with an average power of $250 \mu \mathrm{W}$ at the focus. Moreover, at these low intensities no bleaching occurred, so that one could constantly monitor the $z$-response of the two-photon excitation microscope over tens of minutes.

For the above numerical aperture (1.4) and pixel dwell time $(2 \mathrm{~ms})$, the apparent signal quality of the $z$-response was almost as good as from a mirror. This is exemplified in Fig. 4(b) showing an $x z$ image through the fluorescent layer. The image size was approximately $5 \times 5 \mu \mathrm{m}$. Figure $4 \mathrm{c}$ displays the intensity profile obtained by a single axial scan of the layer through the focus, i.e. the $z$-response. The FWHM of the z-response is about $540 \mathrm{~nm}$ which is in good agreement with data published elsewhere (Schrader et al., 1997).

As a next step we recorded the $4 \mathrm{Pi}$-confocal equivalent, for coherent illumination from both sides (4Pi-confocal of type A), shown in Fig. 5. The location within the sample, the image size and the pixel dwell time were the same as in Fig. 4. The illumination intensity was about $100 \mu \mathrm{W}$, that is the total average power was slightly less than in the single lens recording of Fig. 4 . The intensity profile displays a typical profile of a $4 \mathrm{Pi} z$-response with a sharp maximum of $145 \mathrm{~nm}$ FWHM, and two pronounced lobes of typical height at the given pinhole size. The relative phase between the two
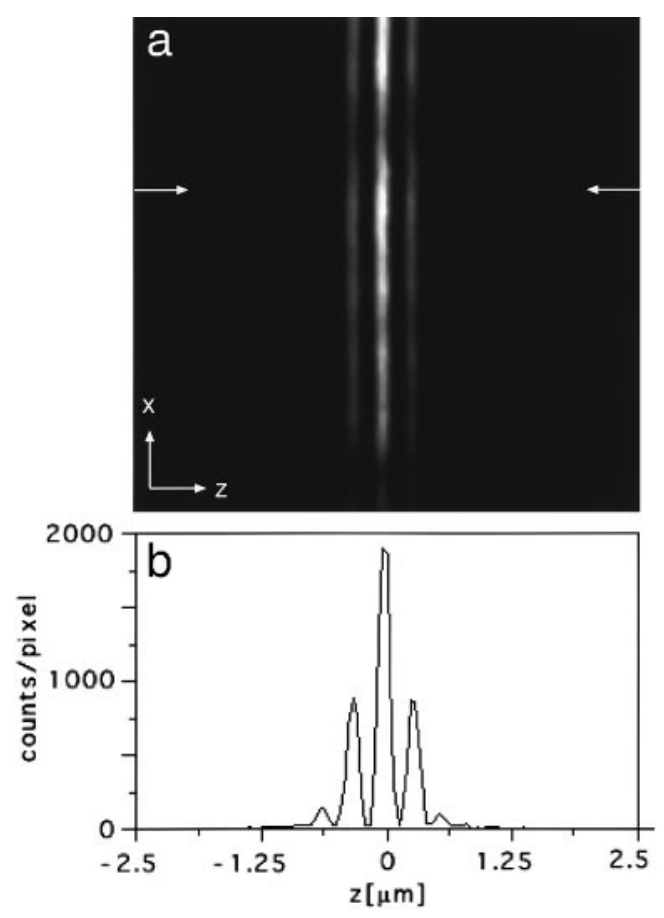

Fig. 5. 4Pi-confocal two-photon excitation $x z$ image of a domain of the EDIPAB film and intensity profile along the optic axis; conditions are as in Fig. 4(b,c). 
interfering illumination beams was controlled by changing the path length of one of the illumination paths with a piezo-electrically driven mirror. In Fig. 5 the relative phase was chosen to be a multiple of $2 \pi$. As in the confocal case, the signal was strong and stable during image acquisition. The layer thickness (a couple of nanometres) is negligible compared with the FWHM of the PSF of the microscope, thus yielding the $z$-response of the microscope. The $z$ responses in Figs. 4 and 5 represent single axial scans; no averaging was used.

Further experiments showed that cultivation of cells onto the EDIPAB-covered coverslips is possible. The layers fulfilled the necessary requirements of mechanical and chemical stability. For instance, it was possible to use them as substrates for growing mouse fibroblast cells. Glycerol and aqueous mounting media did not affect the layers even after several weeks.

\section{Discussion}

Despite being ultrathin, the EDIPAB layers are bright enough to enable the online observation of the axial resolution in one-photon $(488,514,532 \mathrm{~nm}$ excitation) and two-photon $(810,1064 \mathrm{~nm})$ microscopy. We also note that it is not relevant that the layers are monolayers since their thickness is at least two orders of magnitude below the axial focal extent.

As the layers are expected to be chemically compatible with biological samples one could think of using coated coverslips as a standard in 3-D microscopy. In this case, one would obtain the experimental axial resolution jointly with the 3-D image for each $x, y$-coordinate. As the axial FWHM of the fluorescence signal of the film is closely related to the axial FWHM of the PSF (Wilson \& Sheppard, 1984) the $z$ response could be useful for image restoration (Castleman, 1979; Bertero etal., 1990; Shaw, 1995; Van der Voort \& Strasters, 1995; Kano et al., 1996).

When using layered coverslips for biological imaging, the strong fluorescent signal of the EDIPAB layer can be disadvantageous in some cases. The broad emission spectrum (Fig. 2) of these layers counteracts a simple spectral discrimination of the layer fluorescence. We placed biological samples grown on a EDIPAB-layered coverslip into a conventional epifluorescence microscope. Not surprisingly, it was impossible to observe the specimen because the specimen fluorescence was masked by that of the layer, owing to the inability of these systems to discriminate planes. However, we would like to caution that a potential signal overlap might also occur in confocal and multiphoton microscopes when imaging the first $1-2 \mu \mathrm{m}$ below the coverslip. Whereas with one-photon excitation confocal microscopy significant bleaching of the layers is expected, with two-photon excitation this should be of a lesser concern.
For our research on 4Pi-confocal microscopy, EDIPAB layers have virtually replaced the use of thicker dye layer solutions in our laboratory. EDIPAB layers proved very useful in 4Pi-microscopy by facilitating the alignment of the two lenses and defining the relative phase of the interfering wavefronts. Being a subject of research themselves, EDIPAB layers leave considerable room for improvement. An optimization of layer production parameters will probably yield larger homogeneous areas. In that case, preselection of the area on the coverslip will not be required. We also think that the development of fluorescent films with a narrower absorption and emission spectra will be advantageous in many cases. For biological applications it is even possible to envisage chemically modifying the terminal groups of the carbon chains by groups susceptible for binding proteins within cell membranes. Naturally, the success in applying EDIPAB layers encourages us to investigate other ultrathin layers also.

In conclusion, we have demonstrated the use of thin fluorescence layers as an approach for measuring the $z$ response of confocal and two-photon fluorescence microscopes. In contrast to the more conventional method of recording the edge response of thick fluorescence layers, no further derivation of the data is required, so that direct view of the response is possible. Our method is the fluorescence counterpart to the axial scan of a mirror in confocal reflection microscopy. Importantly, the direct observation of spherical aberrations of 3-D fluorescence microscopes allows an interactive system optimization of single-photon confocal and two-photon fluorescence microscopes.

\section{Acknowledgments}

U. G. Hofmann would like to thank J. B. Rosenholm (Åbo Akademi, Turku) for providing the film balance, H. E. Gaub (TU München) for the lipid and J. Peltonen and T. Viitala (Åbo Akademi) for assistance. M. Schrader thanks C. Schnetter (Department of Molecular Biology, MPI Göttingen) for help with the spectrometer and the Zeiss confocal microscope. S. W. Hell acknowledges useful discussions with J. Engelhardt (Leica, Heidelberg). Part of the work was carried out in Finland while the authors were recipients of grants by the European Union.

\section{References}

Bertero, M.P.B., Brakenhoff, G.J., Malfanti, F. \& Van der Voort, H.T.M. (1990) Three-dimensional image restoration and superresolution in fluorescence confocal microscopy. J. Microsc. 157, 3-20.

Born, M. \& Wolf, E. (1993) Principles of Optics, Pergamon Press, Oxford.

Castleman, K.R. (1979) Digital Image Processing. Prentice-Hall, Englewood Cliffs, NJ. 
Denk, W., Strickler, J.H. \& Webb, W.W. (1990) Two-photon laser scanning fluorescence microscopy. Science, 248, 73-76.

Göbel, H.D. (1989) Fluoreszenz-, Röntgen- und Elektronenoptische Untersuchung von Phasenverhalten, Mikrostruktur und Polymerisation monomolekularer Schichten aus vernetzten Lipiden. Doctoral thesis, Technical Unversity, Munich.

Göbel, H.D., Gaub, H.E. \& Möhwald, H. (1987) shape and microstructure of chrystalline domains in polydiacetylene monolayers. Chem. Phys. Lett. 138, 441-446.

Hell, S.W., Reiner, G., Cremer, C. \& Stelzer, E.H.K. (1993) Aberrations in confocal fluorescence microscopy induced by mismatches in refractive index. J. Microsc. 169, 391-405.

Hell, S.W., Utz, A., Hänninen, P.E. \& Soini, E. (1995) Pulsed laser fluorophore deposition: a method for measuring the axial resolution in two-photon fluorescence microscopy. J. Opt. Soc. Am. A, 12, 2072-2076.

Hupfer, B., Ringsdorf, H. \& Schupp, H. (1983) Liposomes from polymerizable phospholipids. Chem. Phys. Lipids, 33, 355-374.

Kano, H., Van der Voort, H.T.M., Schrader, M., van Kempen, G.M.P. \& Hell, S.W. (1996) Avalanche photodiode detection with object scanning and image restoration provides $2-4$ fold resolution increase in two-photon fluorescence microscopy. Bioimaging, 4, 187-197.

Moers, M.H.P., Gaub, H.E. \& van Hulst, N.F. (1994) Polydiacetylenic monolayers studied with a fluorescence scanning near-field optical microscope. Langmuir, 10, 2774-2777.

Schrader, M., Hell, S.W. \& Van der Voort, H.T.M. (1996) Potential of confocal microscopes to resolve in the 50-100 nm range. Appl. Phys. Lett. 69, 3644-3646.

Schrader, M., Bahlmann, K. \& Hell, S.W. (1997) Three-photon excitation microscopy: theory, experiment and applications. Optik, 104, 116-124.

Shaw, P.J. (1995) Comparison of Wide-Field/Deconvolution and Confocal Microscopy for 3D Imaging.' Handbook of Biological Confocal Microscopy, (ed. by J. Pawley), pp. 373-385. Plenum Press, New York.

Sheppard, C.J.R. \& Cogswell, C.J. (1991) Effects of aberrating layers and tube length on confocal imaging properties. Optik, 87, 3438 .

Tillmann, R.W. \& Hofmann, U.G. (1994) AFM-Investigation of the molecular structure of films from a polymerizable two-chain lipid. Chem. Phys. Lipids, 73, 81-89.

Ulmann, A. (1991) An Introduction to Ultrathin Organic Films. Academic Press, San Diego, CA.

Van der Voort, H.T.M. \& Strasters, K.C. (1995) Restoration of confocal images for quantitative analysis. J. Microsc. 178, 165181.

Wilson, T. (1990) Confocal Microscopy. Academic Press, London.

Wilson, T. \& Carlini, A.R. (1989) The effect of aberrations on the axial response of confocal imaging systems. J. Microsc. 154, 243-256.

Wilson, T. \& Sheppard, C.J.R. (1984) Theory and Practice of Scanning Optical Microscopy. Academic Press, New York. 